\title{
Relationship between polymer chain conformation and phase boundaries in a supercritical fluid
}

\author{
Gabriel Luna-Bárcenas, J. Carson Meredith, Isaac C. Sanchez, and Keith P. Johnston \\ Department of Chemical Engineering, University of Texas at Austin, Austin, Texas 78712 \\ Dmitry G. Gromov and Juan J. de Pablo \\ Department of Chemical Engineering, University of Wisconsin, Madison, Wisconsin 53706
}

(Received 3 July 1997; accepted 17 September 1997)

\begin{abstract}
We investigate the solvent density driven changes in polymer conformation and phase behavior that occur in a supercritical fluid, with a particular emphasis on conditions near the lower critical solution temperature (LCST) phase boundary. Using continuous space Monte Carlo simulations, the mean square end-to-end distance $(R)$ and radius of gyration $\left(R_{g}\right)$ are calculated for a single chain with 20 Lennard-Jones segments in a monomeric solvent over a broad range of densities and temperatures. The chains collapse as temperature increases at constant pressure, or as density decreases at constant temperature. A minimum in $R$ and $R_{g}$ occurs at a temperature slightly above the coil-to-globule transition temperature (C-GTT), where the chain adopts a quasi-ideal conformation, defined by the balance of binary attractive and repulsive interactions. Expanded ensemble simulations of finite-concentration polymer-solvent mixtures reveal that the LCST phase boundary correlates well with the single chain C-GTT. At temperatures well above the LCST, the chain expands again suggesting an upper critical solution temperature (UCST) phase boundary above the LCST. (C) 1997 American Institute of Physics. [S0021-9606(97)50548-4]
\end{abstract}

\section{INTRODUCTION}

Polymer chains in solution exhibit various conformational behaviors depending on solvent conditions. In a " $\mathrm{good}$ solvent," the polymer adopts a random coil (expanded) conformation, whereas in a "poor solvent" and in dilute solution the polymer chain collapses, producing a globular conformation. In a nondilute solution, where on average chains overlap with one another, chains adopt expanded coil-like conformations in both good and poor solvents. However, in poor solvents, the nondilute solution can split into a polymer-rich phase and a polymer-depleted phase.

The first experimental evidence for a coil-to-globule transition was probably the denaturation of proteins on heating. The biologically active state of proteins corresponds to a compact (globule) state. When the protein is heated, the chain unfolds into a coillike architecture in which its biological activity is reduced. ${ }^{1}$ As early as 1960 , Stockmayer ${ }^{2}$ first pointed out that a chain molecule must collapse to a rather dense form if the net attraction between its monomers becomes sufficiently large. Since then, a number of theoretical and computational studies on the coil-to-globule transition have appeared in the literature. ${ }^{3-5}$ These studies were traditionally associated with lowering temperature to approach an upper critical solution temperature (UCST) phase boundary in dilute solutions. On the experimental side, there has been a great deal of work on the dynamic and static properties of dilute polymer solutions in good, theta, and poor solvents near the UCST. ${ }^{1,6}$

As is well-known, polymer solutions also exhibit lower critical solution temperature (LCST), or thermally induced, phase separation where phase splitting is induced by raising temperature. LCSTs have been observed in strongly interacting polar mixtures, for example aqueous solutions, as well as in weakly interacting nonpolar polymer solutions. A LCST may be produced by strong specific interactions among components and/or by the mixture's finite compressibility. Unlike the UCST, which is driven by unfavorable energetics, a thermodynamic analysis shows that the LCST is an entropically driven phase separation. ${ }^{7}$ In nonpolar polymer solutions compressibility effects are dominant, and both the FloryOrwoll-Vrij-Eichinger (FOVE) (Ref. 8) and the SanchezLacombe or Lattice-Fluid (LF) (Ref. 9) equations of state adequately describe the LCST behavior. It has also been shown that a LCST is associated with large size differences between a polymer and a solvent. ${ }^{10}$ For instance, the classical Flory-Huggins theory, ${ }^{11}$ which ignores differences in the equation of state properties of the pure components, completely fails to describe the LCST behavior.

In polymer-solvent mixtures the existence of a LCST is the rule, not the exception. ${ }^{7}$ It was conjectured some time ago that chain collapse should be observed near a LCST in an analogous way as it occurs near an UCST. ${ }^{12}$ The LCST usually occurs in the vicinity of the vapor-liquid critical temperature of the pure solvent $(0.7-0.9$ of the pure solvent critical temperature). LCST phase behavior is often observed for polymer solutions in supercritical fluids (SCFs). ${ }^{13-16}$ To our knowledge, neither experimental nor theoretical studies of polymer chain collapse near the phase boundary associated with the LCST have yet been reported.

The development of a fundamental understanding of the polymer conformations and phase behavior of polymer solutions in SCFs is a theoretical challenge of great practical interest. The solution behavior is complex due to the large values of free volume, isothermal compressibility, volume expansivity, and concentration fluctuations. Practical SCF applications involving LCST phase behavior include poly- 
mer fractionation, impregnation and purification, polymer extrusion and foaming, formation of materials by rapid expansion from supercritical solution and precipitation with a compressed fluid antisolvent, dispersion and emulsion polymerization, and the formation of microemulsions and emulsions. ${ }^{17-19}$ McClain et al. ${ }^{20}$ used neutron scattering to determine the phase behavior and chain dimensions of dilute solutions of poly(1,1-dihydroperfluorooctyl acrylate) in SCF carbon dioxide $\left(\mathrm{CO}_{2}\right)$ as a function of pure $\mathrm{CO}_{2}$ density (solvent quality). This system is known to exhibit LCST behavior. The second virial coefficient of the polymer was positive for the high $\mathrm{CO}_{2}$ densities studied. In the future, it would be interesting to examine changes in the second virial coefficient and polymer chain dimensions as density is lowered.

Homopolymers and block- and graft-copolymers have been used as stabilizers to form microemulsions, emulsions, and latexes in supercritical fluids. ${ }^{21,22}$ In the copolymers, one of the blocks is " $\mathrm{CO}_{2}$-philic." It is important to know the conformation of this block in $\mathrm{CO}_{2}$ as well as the phase behavior near the LCST, since these properties play an important role in colloid stability.

In recent years, new Monte Carlo methods for computer simulation of polymeric systems have appeared in the literature. ${ }^{23-25}$ The continuum configurational bias (CCB) method has reduced the CPU time usage by several orders of magnitude compared to the simple reptation algorithm. ${ }^{24}$ With the advent of new powerful simulation techniques, a number of interesting problems can now be investigated. Examples include direct simulation of phase equilibria in homopolymers ${ }^{26,27}$ and systems with many chains or at high density using expanded ensemble techniques. ${ }^{28}$ We recently reported in a letter the conformational properties of a single polymer chain of length 20 segments in a monomeric solvent near the LCST. ${ }^{29}$ This previous work was, to our knowledge, the first simulation study of polymer conformation and phase behavior near the LCST. The chain collapses with decreasing solvent density in the attractive (Lennard-Jones) system due to the loss of solvent screening of intrachain interactions. In contrast, excluded volume interactions cause chain expansion in an athermal system. ${ }^{30}$ For the Lennard-Jones system, a qualitative relationship was proposed between the coil-toglobule transition temperature (C-GTT) and the LCST along an isobar. In the present work, this relationship is determined quantitatively.

The overall objective of this work is to study the underlying physics of polymer chain conformation and solventpolymer phase behavior, over a wide range in temperature and solvent density. A key question is whether the conformational properties of a single chain may be used to predict the location of the LCST phase boundary for a finiteconcentration solution. We hypothesize that the LCST of a finite-concentration mixture is near the C-GTT for a single chain. This hypothesis is tested with direct simulations of phase separation as a function of solvent density using the expanded Gibbs (EG) ensemble method. ${ }^{28}$

In the next two sections the molecular model and simulation method are explained. Then, the dimensions of single chains of size $N=20$ and 40 are investigated in vacuum to serve as a background for understanding the ensuing studies of solvent effects. In the absence of solvent, the dimensions of an isolated chain are determined by both energetic and entropic (self-excluded volume) forces, and upon cooling, a coil-to-globule transition occurs at a unique temperature (CGTT). In the next section, solvent molecules are added to the system, in contrast to previous studies of chain conformation in vacuum. Solvent molecules must be included to describe the large difference in polymer and solvent equation of state properties that govern the LCST in compressible polymer solutions. The system is symmetric in that solvent and polymer segments are identical, i.e., $\epsilon_{11}=\epsilon_{22}=\epsilon_{12}$ (equal energetics) and $\sigma_{11}=\sigma_{22}=\sigma_{12}$ (equal sizes), where $\epsilon$ and $\sigma$ are the energy and diameter.

The C-GTT is determined for a wide range of solvent densities in the gas, supercritical fluid, and liquid states at the point where a single chain adopts a quasi-ideal conformation (pairwise attractive forces balance the repulsive forces). The C-GTT is presented using temperature-density, pressuredensity, and pressure-temperature diagrams and compared with simulations of the LCST. Furthermore, based upon single chain conformations, we predict the occurrence of an UCST at temperatures above the LCST. The predicted values of the LCST and UCST from chain conformation provide useful starting points in searching for phase separation by the direct method.

\section{MOLECULAR MODEL}

The systems studied in this work consist of a single freely jointed chain (chain length $N=20$ ) immersed in a medium of solvent molecules. The energetic interactions are modeled via the Lennard-Jones (L-J) potential, which is given by

$$
U_{i j}(r)=\left\{\begin{array}{l}
4 \epsilon_{i j}\left[\left(\frac{\sigma_{i j}}{r}\right)^{12}-\left(\frac{\sigma_{i j}}{r}\right)^{6}-\left(\frac{\sigma_{i j}}{r_{c}}\right)^{12}+\left(\frac{\sigma_{i j}}{r_{c}}\right)^{6}\right] \quad r \leqslant r_{c} \\
0 \quad r>r_{c}
\end{array}\right.
$$

where $r$ is the site-site distance, and the L-J potential only operates to distances up to $r_{c}=2.5 \sigma$ (the cutoff). For separations larger than $r_{c}$ the potential is defined to be zero. The terms containing $r_{c}$ in Eq. (1) shift the potential upward to assure continuity at the cutoff. Three types of interactions are considered; segment-segment for polymer sites that are not bonded, segment-solvent, and solvent-solvent. The bond lengths are held constant at $1 \sigma$.

The phase and critical behavior of this L-J model has been characterized for simple monomeric fluids by Johnson, ${ }^{31}$ Smit, $^{32}$ and Gromov. ${ }^{33}$ The latter reports a reduced critical temperature of $T_{c}^{*}=k T_{c} / \epsilon=1.08$, a reduced critical density of $\rho_{c}^{*}=\sigma^{3} \rho_{c}=0.31$, and a reduced critical pressure of $P_{c}^{*}=P_{c} \sigma^{3} / \epsilon=0.10$. 


\section{SIMULATION METHODOLOGY}

\section{A. Canonical ensemble simulations}

In this work the continuum configurational bias (CCB) Monte Carlo method was used to characterize polymer chain conformation. The method consists of cutting the chain at some randomly selected site. A portion of the chain is deleted from this point to one of the ends. The chain is then regrown site by site until its original length is restored. For each site to be regrown, $N_{\text {samp }}$ trial orientations of the connecting bond vector are generated. (In our study $N_{\text {samp }}=6$.)

For flexible chains the possible positions of each appended site are sought uniformly on a spherical surface centered at the previous existing site. This way of selecting the new site position favors nonoverlapping, low-energy configurations and thus introduces a bias that must be removed to ensure microscopic reversibility. This is accomplished by introducing the appropriate weights into the acceptance/ rejection criterion of the proposed move. Details on the computer implementation of the CCB method and its capabilities and limitations have been discussed elsewhere. ${ }^{24,34}$

The above CCB prescription is then applied in the canonical $(N V T)$ ensemble formalism. The variables are reduced in the usual manner; system temperature, $T^{*}$ $=k_{B} T / \epsilon$, and density, $\rho^{*}=\sigma^{3} \rho$, with $\rho=N_{S} / V$, where $N_{S}$ is the number of solvent molecules. In this work $T^{*}$ is varied from 0.7 to 5.0 and $\rho^{*}$ is varied from 0.0 to 0.65 . Extremely dilute solutions are necessary when investigating chain conformation to avoid interpenetration and competitive effects, such as chain aggregation and phase transitions that are associated with more concentrated solutions. Thus the box length was chosen to be $L \geqslant 15.0 \sigma(>N / 2)$ to avoid any selfinteraction between chain sites through the periodic boundary conditions. The number of solvent molecules and the box length, $L$, were selected to achieve the desired system density. For example, for $\rho^{*}=0.3$ and $L=15.0 \sigma$, the number of solvent segments is $N_{s}=\rho^{*} L^{3}=1012$. The number of solvent molecules varied from 0 to 1670 for $0 \leqslant \rho^{*} \leqslant 0.50$.

For $\rho^{*} \geqslant 0.5$ the CCB method becomes highly inefficient (successful moves are scarce), and the multiple time step hybrid Monte Carlo $^{33}$ technique was used. This hybrid method was also employed at lower densities to double check the CCB results for the end-to-end distance. The results were in good agreement for reduced densities from 0 to 0.5 at reduced temperatures from 1.1 to 2.0. Relaxation of the solvent was achieved by random displacement Monte Carlo moves. Statistics for conformational properties, i.e., mean square end-to-end distance, $\left\langle R^{2}\right\rangle$, and mean square gyration radius, $\left\langle R_{g}^{2}\right\rangle$, were collected for $O\left(10^{7}\right)$ steps after an equilibration period of at least $5 \times 10^{6}$ steps. This recipe gives $O\left(10^{6}\right)$ successful chain moves. Average run times on the Cray J90 at the University of Texas at Austin varied from about 10 to $80 \mathrm{CPU}$ h for $0 \leqslant \rho^{*} \leqslant 0.50$.

\section{B. Expanded Gibbs ensemble simulations}

A number of methods for simulating phase equilibria of polymeric systems have appeared in the literature. ${ }^{24-26,35-37}$

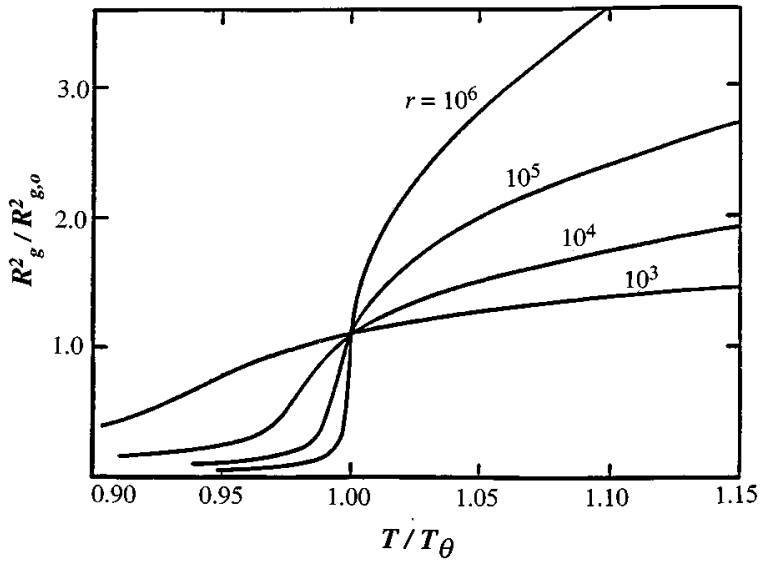

FIG. 1. Schematic of the normalized mean square gyration radius $\left(R_{g, 0}^{2}\right.$, ideal reference dimensions) as a function of system temperature in vacuum for various polymer molecular weights $(r)$. Taken from Ref. 12

However, all of these methods become highly inefficient at high system densities. To circumvent this problem, Escobedo and de $\mathrm{Pablo}^{28}$ recently extended the Gibbs ensemble method $^{26}$ in an expanded ensemble formalism (EG). The idea behind the EG method is to efficiently sample the configurational space, especially for dense systems and for large, complex molecules, by molecular creation and destruction of a tagged chain of variable length. This new EG method allows one to directly simulate phase equilibria of chainlike molecules with very fast equilibration. Details of this novel method are given elsewhere. ${ }^{28}$ In this work the EG method was employed to study polymer-solvent phase boundaries. The systems studied were composed of 25 chains $(N=20)$ and 1920 solvent molecules at reduced pressures, $P^{*}$, of 0.15 and 0.20 . Thus, the overall polymer composition was about 0.2 (weight or occupied volume fraction) for all runs. Various molecular moves were used to obtain phase equilibria information, including multiple time step hybrid $\mathrm{MC}$ moves, ${ }^{33}$ volume moves ( 6 per cycle), Gibbs transfer moves (1500 attempted per cycle), and EG ensemble moves. ${ }^{28}$ Typical runs consisted of a total of 10000 cycles.

\section{RESULTS AND DISCUSSION}

\section{A. Chain dimensions at zero density}

Chain collapse when lowering the temperature, associated with UCST behavior, is depicted in Fig. 1. The normalized gyration radius of an isolated chain in vacuum is plotted as a function of temperature for different polymer molecular weights. ${ }^{12}$ Even though the polymer chain is in a vacuum, the solvent quality is emulated by manipulating the temperature. In such systems the strength of the attractive interactions is proportional to $\epsilon / k T$, i.e., at high enough temperatures $\epsilon / k T \rightarrow 0$, and the chain will expand (good solvent regime); at low temperatures the chain will collapse. The no solvent, or vacuum, treatment has been used to ease the calculation in most previous simulations. Typical experimental data for polystyrene in cyclohexane solutions ${ }^{38}$ shows exactly the same behavior as in Fig. 1. 


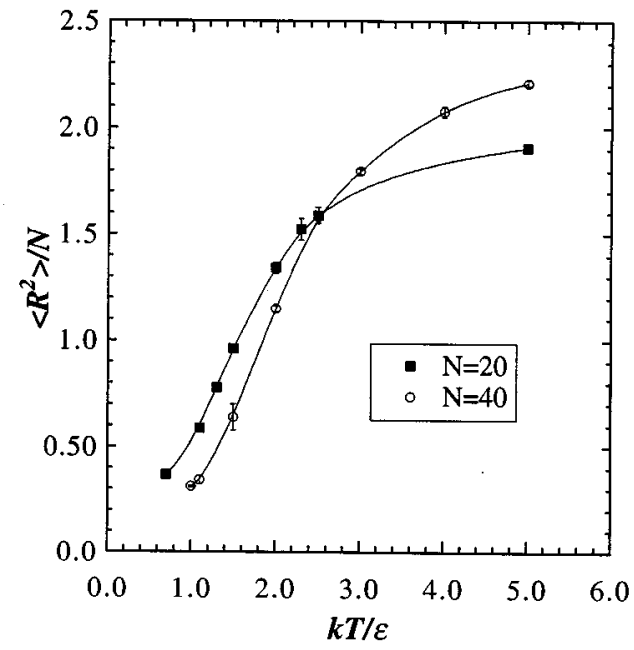

FIG. 2. Normalized mean square end-to-end distance of a single polymer chain $(N=20,40)$ in vacuum as a function of system temperature

A common feature of the chain behavior in Fig. 1 is that all molecular weight curves intersect at a unique temperature. This temperature is defined as the coil-to-globule transition temperature (C-GTT) in vacuum. For an infinite molecular weight polymer, the C-GTT becomes the $\theta$ temperature, where the chain behaves quasi-ideally. ${ }^{1}$ Here, pairwise attractive and repulsive interactions compensate. For a binary polymer-solvent mixture, the $\theta$ temperature defines the limiting UCST for a polymer of infinite molecular weight.

The $\theta$ temperature is an important thermodynamic parameter since it distinguishes two regions in a dilute polymer-solvent phase diagram. Above $\theta$ the polymer adopts coil-like conformations (good solvent), whereas below $\theta$ the polymer will begin to collapse. Another significant property of $\theta$ is that it separates the regimes of small fluctuations (below $\theta$ ) and large fluctuations (above $\theta$ ) in chain dimensions. $^{3} \theta$ is also an intrinsic property of a particular system, i.e., different potential functions will produce different $\theta \mathrm{s}$. Therefore, it is important to characterize the C-GTT in vacuum for our model system.

Figure 2 shows the chain behavior in vacuum for the L-J potential shown in Eq. (1). The normalized mean square endto-end distance $\left\langle R^{2}\right\rangle / N$ of a single chain is plotted as a function of system temperature for two chain lengths $(N=20$, 40). From this graph, the C-GTT in vacuum is about 2.5 (intersecting point). This calculation is of great importance to place in perspective the effect of solvent density on chain conformation and phase behavior.

\section{B. Solvent density effect on chain dimensions}

\section{Thermodynamic stability}

From the classical Flory-Huggins theory it is known that polymeric mixtures are much less miscible than those of smaller molecules due to the much smaller entropy of mixing. According to the Flory-Huggins model a binary system is stable to any concentration fluctuation if ${ }^{11}$

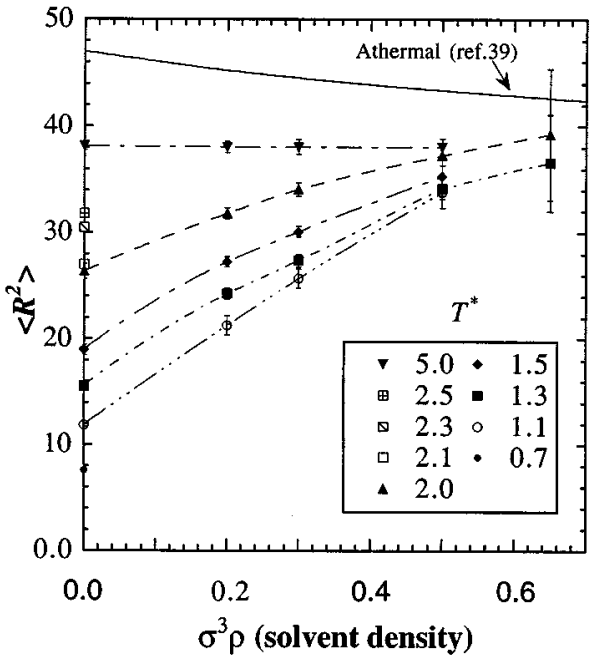

FIG. 3. Isotherms of the mean square end-to-end distance of a single polymer chain $(N=20)$ as a function of solvent density. The pressure along an isotherm increases with increasing density.

$$
\left.\frac{\partial^{2} \Delta A_{\text {mix }}}{\partial \phi^{2}}\right)_{T, V}=\frac{1}{N_{1} \phi}+\frac{1}{N_{2}(1-\phi)}-2 \chi>0,
$$

where $A_{\text {mix }}$ is the Helmholtz free energy of mixing, $\phi$ is the volume fraction of one of the components, and $N_{i}$ is the chain length of component $i$. The Flory parameter, $\chi$, is given classically by

$$
\chi \propto \frac{\epsilon_{11}+\epsilon_{22}-2 \epsilon_{12}}{k_{B} T} .
$$

In a mixture with symmetric energetics $\left(\epsilon_{11}=\epsilon_{22}\right.$ $\left.=\epsilon_{12}\right) \chi \rightarrow 0$. It follows from Eq. (2) that $\left(\partial^{2} \Delta A_{\text {mix }} / \partial \phi^{2}\right)_{T, V}$ will always be positive. Therefore, in the spirit of the FloryHuggins model, a symmetric mixture such as the one described in this work will never experience any phase instability associated with the conventional UCST, which occurs at temperatures below the LCST. It is noteworthy that Eq. (2) has been derived for an incompressible system; it fails to describe LCST behavior. However, when the finite compressibility and large size difference among system components are taken into account, phase stability is drastically affected and now the system is able to explore other equilibrium states including phase separation. To summarize, a symmetric polymer solution was purposely chosen so that the UCST behavior is erased from the phase diagram. This approach allows one to explore any chain conformational changes and possible phase transitions that could be related to LCST behavior, and avoid the above type of UCST behavior. Let us now examine the chain behavior as a function of solvent density.

\section{Chain dimensions}

Figure 3 shows the mean square end-to-end distance, $\left\langle R^{2}\right\rangle$, of the chain as a function of the system density at several reduced temperatures. At high densities, the chain adopts a coillike conformation that approaches the athermal 
or infinite temperature limit. ${ }^{39}$ It should be noted that along an isotherm, the system pressure varies. The chain collapse at low densities suggests that solvent quality diminishes as the solvent density decreases. This result is opposite to that observed in systems without attractive interactions. ${ }^{30,40}$ In pure repulsive systems, an isolated polymer chain is expanded at low solvent densities and its collapse is promoted by increasing solvent density, i.e., solvent quality decreases with increasing solvent density (or equivalently, increasing pressure). In the absence of attractive interactions, the driving force for chain collapse is a chain-solvent excluded volume effect. ${ }^{30}$ As Fig. 3 shows, the presence of attractive interactions qualitatively changes the dependence of solvent quality on density. Attractive interactions seem to dominate any excluded volume effects and completely change the dependence of solvent quality on density. But why? The rest of this work addresses this question.

In the absence of solvent, i.e., in a vacuum, the dimensions of an isolated polymer chain are determined by two opposing forces; ${ }^{12}$ intrachain attractive forces act to collapse the chain to a globular form, whereas entropic forces act to keep the chain expanded in random coil-like conformations. Upon cooling a coil-to-globule transition occurs at a unique critical temperature (C-GTT) where intrachain attractive forces overcome the entropic force. From Fig. 2, the vacuum value for the C-GTT is about 2.5. (See Sec. IV A.) Adding solvent depresses the C-GTT relative to its vacuum value. The primary effect of solvent is to mediate the intrachain attractive forces. The solvent "screens" the intrachain attractive interactions in the following sense: the presence of solvent molecules within the polymer chain domain impedes collapse by excluding close intrachain contacts (a solvent excluded volume effect). The denser the solvent, the more effective it is in screening attractive intrachain forces. For example, in Fig. 3, note that the chain dimensions are all shifted upward as the solvent density increases, and the isotherms begin to merge. The merging and rising of the isotherms indicate that the strength of intrachain attractive interactions is being effectively screened by the solvent.

Note also from Fig. 3 that $\left\langle R^{2}\right\rangle$ increases monotonically with temperature. The increase in chain dimensions with temperature at constant density (pressure must also increase to maintain constant density) is consistent with the idea that attractive energetics become less important at high temperatures $(\epsilon / k T \rightarrow 0)$. In the extreme high temperature limit, where attractive forces are completely ineffective and where the solvent density approaches zero, the self-excluded volume intrachain interaction dominates and the chain reaches its maximum expansion (athermal limit). But there is much more to this story.

When the chain collapses to enhance favorable intrachain attractive interactions, it does so at the expense of losing chain conformational entropy. Chain connectivity brings chain segments into close proximity to one another (the socalled correlation hole), enhancing the effects of intrachain attractive forces relative to chain-solvent interactions. The full range and remarkable dependence of the chain dimensions on temperature is illustrated in Fig. 4. At constant pres-

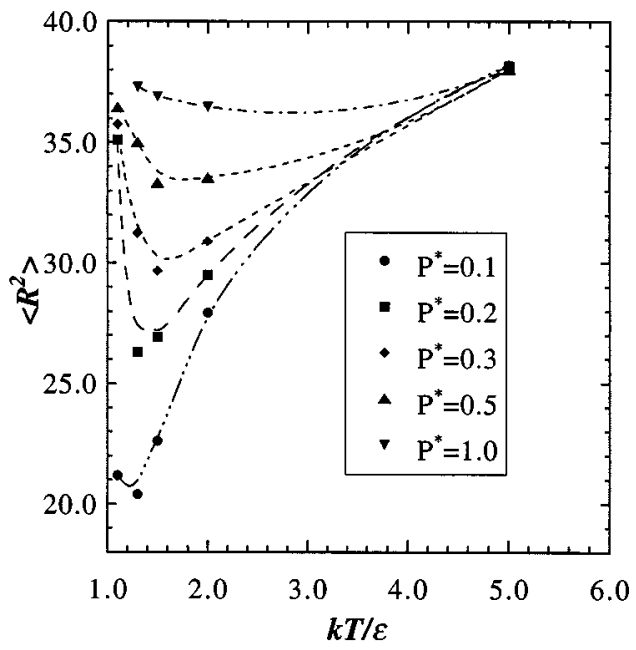

FIG. 4. Mean square end-to-end distance as a function of system temperature at several isobars. See text and Fig. 7 for an explanation of the minimum near the LCST. Data shown are interpolations from Fig. 3.

sure, chain dimensions go through a minimum, suggesting that a phase boundary is being approached. It is possible that this boundary is related to a LCST since the chain collapses when temperature is raised. Upon heating, the chain begins to expand again, implying that solvent quality is improving. Here, the solvent density is very low. This chain expansion suggests that we may encounter a one phase region at temperatures well above the LCST!

Note from Fig. 4 that for $P^{*}>0.5,\left\langle R^{2}\right\rangle$ changes little with temperature. Without much chain collapse, phase separation is not expected. These results suggest that there might be a limiting pressure for a closed immiscibility loop in the phase diagram, and this will be shown below. As $P^{*}$ is decreased, chain collapse becomes more pronounced.

\section{Solvent density effect on C-GTT}

An important parameter that describes whether a chain behaves quasi-ideally (no binary excluded volume interactions) is $\mathfrak{N}=\left\langle R^{2}\right\rangle / 6\left\langle R_{g}^{2}\right\rangle$, where $\left\langle R^{2}\right\rangle$ is the end-to-end distance and $\left\langle R_{g}^{2}\right\rangle$ is the gyration radius. Throughout this paper we distinguish between quasi-ideal chains where the second virial coefficient is zero, and completely ideal chains where all virial coefficients are zero. For a quasi-ideal chain in the limit of infinite chain length, des Cloizeaux and Jannink ${ }^{41}$ obtained $\mathfrak{N}=1$. For chains with excluded volume interactions (self-avoiding chains), Sanchez ${ }^{42}$ predicted $\mathfrak{N}=1.07$ with a pseudo-Gaussian model. Computer simulation studies $^{43}$ show $\mathfrak{N}=1.06$ and renormalization techniques ${ }^{44}$ give $\mathfrak{N}=1.05$. Experimental data of partially deuterated polystyrene in a good solvent $\left(\mathrm{CS}_{2}\right)$ yield ${ }^{45} \mathfrak{N}=1.05$, in good agreement with the theoretical predictions above. The important issue here is that a real chain is expanded relative to its quasi-ideal dimensions due to excluded volume effects. des Cloizeaux and Jannink ${ }^{41}$ predict $\mathfrak{N}=1 / 3$ for the collapsed state of an isolated chain in a poor solvent. In summary, three regimes can be identified for a polymer solution in 


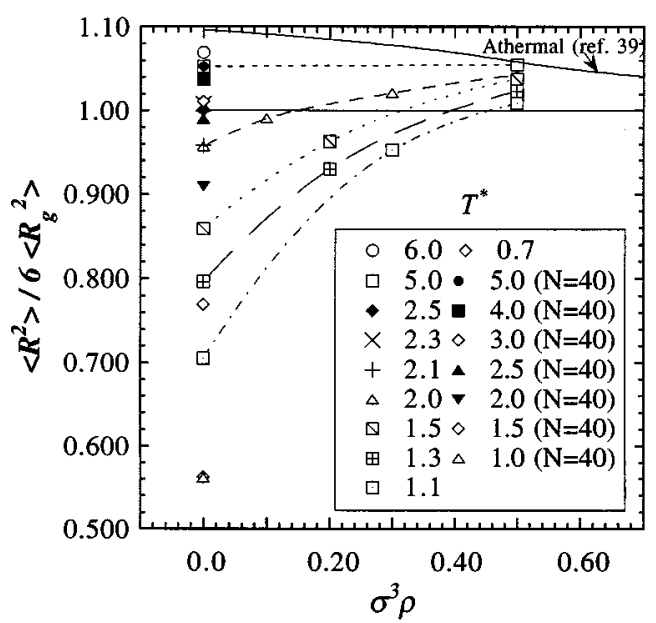

FIG. 5. $\mathfrak{N}$-behavior as a function of solvent density. $\mathfrak{N}=1$ denotes ideal polymer behavior (attractive and excluded volume interactions are balanced). Different isotherms are shown for $N=20,40$. However, for $N=40$ only zero-density data are shown. See text for a detailed explanation of $\mathfrak{N}$.

terms of $\mathfrak{N}$; for real polymers in good solvents $\mathfrak{N}$ $=1.05-1.07$, for quasi-ideal chains $\mathfrak{N}=1$, and for a collapsed chain $\mathfrak{N}=1 / 3$. This analysis serves as a basis to put our results in perspective.

Figure 5 shows the simulation results for the behavior of $\mathfrak{N}$ as a function of solvent density for various temperatures. Two chain lengths are investigated, $N=20,40$; however, for $N=40$ results are only shown when the chain is in a vacuum, i.e., zero solvent density. These results access all three of the above polymer regimes. For the lowest temperatures and at zero density $\left(T^{*}=0.7\right.$ for $N=20$ and $T^{*}=1.0$ for $\left.N=40\right)$, $\mathfrak{N} \sim 0.54$, which is relatively close to the theoretical prediction of $\mathfrak{N}=1 / 3$ for a fully collapsed chain. It is important to mention that this theoretical value $(\mathfrak{N}=1 / 3)$ was obtained in the limit of an infinite chain length. We do not reach the asymptotic theoretical prediction primarily because of the finite size of our chains. One expects that increasing chain size at low temperature will eventually lead to the $\mathfrak{N}=1 / 3$ limit.

An interesting feature observed at high densities is the merging of all isotherms, indicating that the intrachain interactions are being mediated by the solvent. This observation is in agreement with Fig. 3 as previously discussed. Note also that at sufficiently high densities $(>0.50)$ all isotherms will likely merge into a common point that approaches the asymptotic athermal limit. From Fig. 5, it is estimated that the asymptotic behavior will be reached at $\mathfrak{N} \sim 1.05$. This asymptote is the limiting case for a swollen chain (a chain with excluded volume interactions) as discussed above.

Note from Fig. 5 that all isotherms in the range of 0.7 $<T^{*}<2.0$ intersect the quasi-ideal behavior line $(\mathfrak{N}=1)$ at a particular solvent density. The temperature-density locus where $\mathfrak{N}=1$, which describes the quasi-ideal chain behavior, represents the depressed density-dependent C-GTT. From Fig. 5 the C-GTT $(\mathfrak{N}=1)$ at zero density is about 2.5 \pm 0.2 , which is in excellent agreement with the vacuum $\mathrm{C}$ -

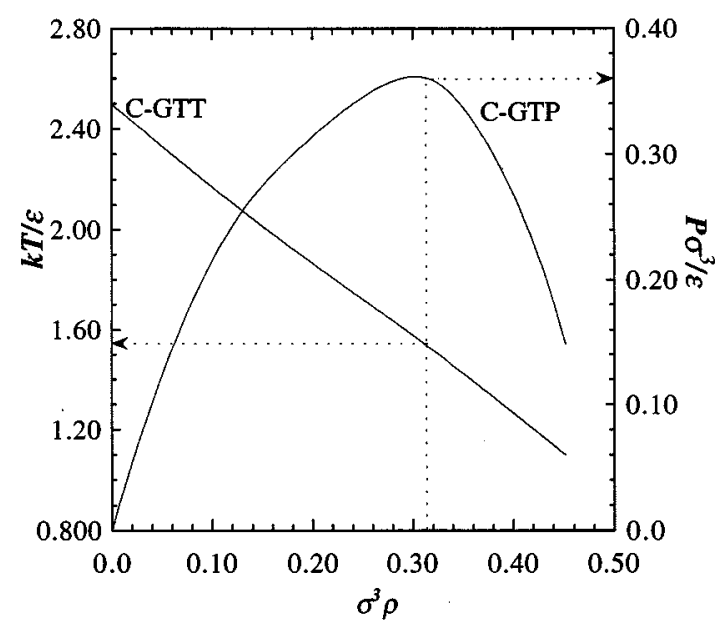

FIG. 6. Coil-to-globule transition temperature (C-GTT) and C-GT pressure (C-GTP) as a function of solvent density. Note that the C-GTT is linearly depressed from its value in vacuum as solvent density increases. The C-GTT is expected to be insensitive to pressure and only a function of solvent density and chain size. The C-GTP shows a maximum at $P^{*}=0.36$. See text and Figs. 7-9 for an explanation of the maximum.

GTT estimation from Fig. 2 by a separate technique. A temperature-density (or pressure-density) plot for $\mathfrak{N}=1$ can now be constructed to analyze the C-GTT behavior. A theta temperature of 3.0 was reported for longer chains $(32<N$ $<400$ ) using a similar model, which included an additional Hookean spring potential between bonded segments. ${ }^{46}$ Our C-GTT is different because bond lengths are rigid in this study and perhaps because of fluctuations associated with shorter chains.

The behavior of the C-GTT with solvent density is illustrated in Fig. 6. The pressure for each density and C-GTT is also shown. The initial effect of solvent is to lower the CGTT relative to its value in vacuum (2.5) by the screening mechanism mentioned earlier. Note that an elegantly simple response is observed; the C-GTT is depressed nearly linearly as the solvent density is increased. The C-GTT line is not expected to be related linearly to pressure since the screening effect depends directly upon solvent density. The pressure behavior is qualitatively different; it shows a maximum at $P^{*} \approx 0.36$ for $\rho^{*} \approx 0.32$ that corresponds to $T^{*} \approx 1.53$. This seemingly complicated behavior is now discussed.

In order to predict phase behavior from the C-GTT data, an equation of state is needed for the polymer solution. For an extremely dilute solution, the equation of state is essentially that of the pure solvent. Figure 7 shows the C-GTT behavior in the temperature-density plane and various isobars (dashed lines) for the pure monomeric L-J solvent obtained with the Johnson equation of state. ${ }^{31}$ Note that the solvent isobars below $P^{*}=0.36$ cross the C-GTT curve at two points, which indicates the existence of a LCST at low temperatures and an UCST at higher temperatures, forming a closed immiscibility loop in the phase diagram as discussed above. This closed loop behavior is illuminated by the $\left\langle R^{2}\right\rangle$ values in Fig. 4 where, starting at low temperature, the chain is highly expanded. As temperature is increased $\left\langle R^{2}\right\rangle$ de- 


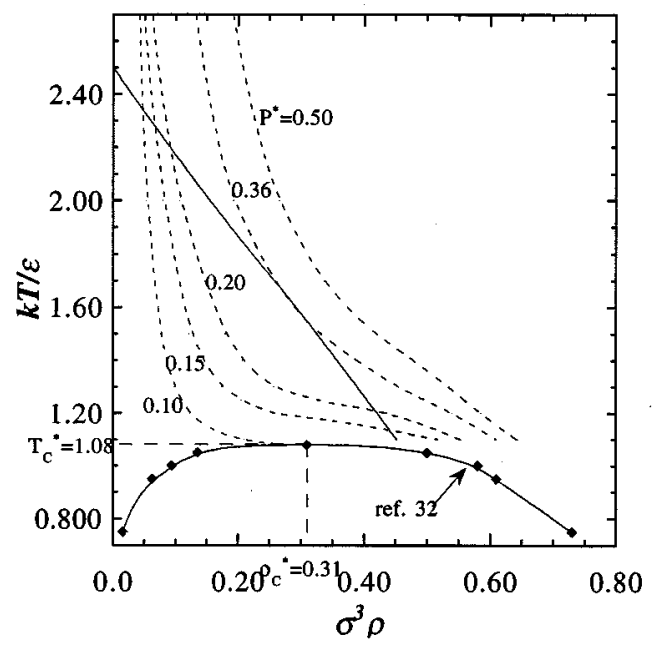

FIG. 7. Variation of the C-GTT with solvent density (solid line). Dotted lines are pure solvent isobars. This figure suggests the existence of an UCST above the LCST (closed immiscibility loop) which will eventually vanish at high enough pressures, since the solvent isobar will lie completely above the C-GTT line and no chain collapse will be observed.

creases, reaching a minimum at a temperature a little above the LCSTs predicted by Fig. 7. In this region, a small increase in temperature produces a large reduction in density and thus causes chain contraction. However, as temperature is increased further, the chain expands again as the interaction between chain segments becomes weaker relative to $k T$. Note also in Fig. 7 that as pressure increases, the isobar moves away from the C-GTT line, and at sufficiently high pressures no collapse transition is observed. Note that the 0.36 isobar is tangent to the C-GTT curve; it sets the maximum pressure where the closed immiscibility loop is observed, i.e., at $P^{*}>0.36$ the system exists as one single homogeneous phase. Note also that $P^{*}=0.36$ corresponds to the maximum in C-GTP observed in Fig. 6. Note that Fig. 4 is in agreement with the above observation: at higher pressures chain collapse is less severe, i.e., at $P^{*}>0.36$ the chain dimensions vary much less with temperature than at lower pressures.

In Fig. 8 the behavior of the C-GT pressure (C-GTP) is shown along with several pure solvent isotherms. From this figure a number of isotherms in the range of $1.1<T^{*}<2.5$ intersect the C-GTP curve analogous to the behavior in Fig. 7. These intersections define the LCST and UCST branches. Intersection points in the range of $1.1 \leqslant T^{*}<1.53$ (low temperature) define the LCST branch, while those where 1.53 $<T^{*}<2.5$ define the UCST branch (high temperature, low density). The $T^{*}=2.5$ solvent isotherm is tangent to the $\mathrm{C}$ GTP curve; it defines the maximum UCST for the system, i.e., at $T^{*}>2.5$ the system coexists as one single homogeneous phase for any pressure (see Fig. 6).

With the aid of Figs. 7 and 8 a pressure-temperature representation of the C-GT may be constructed as shown in Fig. 9. Note that the maximum $\left(T^{*}=1.53, P^{*}=0.36\right)$ denotes the vanishing point of the closed immiscibility loop. A similar $P-T$ diagram was discussed previously in a number

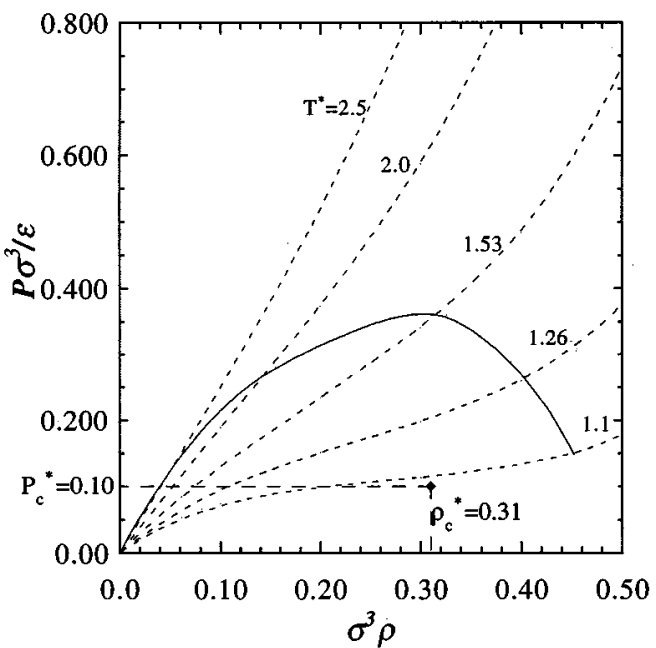

FIG. 8. C-GT pressure (C-GTP) as a function of solvent density (solid line). Dotted lines are pure solvent isotherms. Note that the 2.5 isotherm is tangent to the C-GTP indicating the maximum temperature to observe an UCST. Temperatures above 2.5 will lie completely outside the C-GTP and no UCST will be predicted.

of studies of polymers in supercritical fluid mixtures. ${ }^{13,16}$ However, the $P-T$ behavior shown in Fig. 9 is unique in the sense that the usual UCST branch (UCST below the LCST) is not present. Note that this usual UCST critical line has been erased from the phase diagram due to the symmetric nature of our polymer solution.

Figure 7 predicts that for any $P^{*}<0.10$ the UCSTs observed at high temperatures (intersection of isobar and CGTT) approach that of the C-GTT in vacuum $\left(T^{*}=2.5\right)$. Consequently, the highest predicted UCST is about 2.5. On the other hand, Fig. 7 predicts no LCSTs for $P^{*}<0.10$, i.e., the intersection of an isobar with the C-GTT curve occurs within the two-phase vapor-liquid curve for the pure solvent

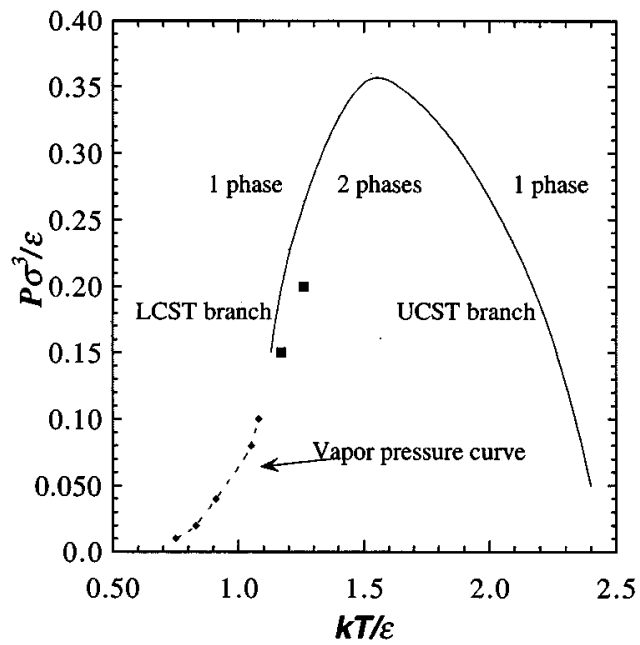

FIG. 9. Pressure-temperature polymer solution behavior predicted from single chain collapse information (infinitely dilute polymer solution). Filled square symbols are results from the direct phase equilibria simulations for a finite-concentration polymer solution. 


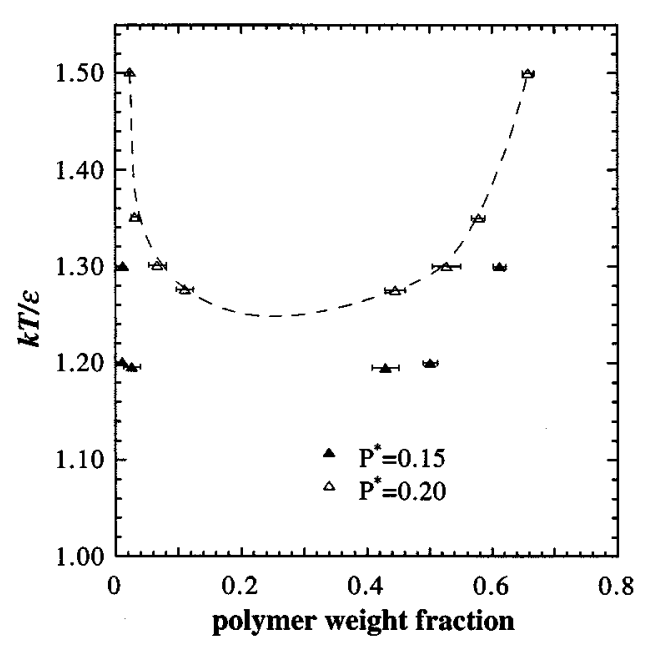

FIG. 10. Temperature-polymer weight fraction phase diagram. LCST $\sim 1.26$ at $P^{*}=0.20$ and $\sim 1.16$ at $P^{*}=0.15$.

(metastable region). Also, as previously noted in Fig. 8, the $T^{*}=2.5$ isotherm is tangent to the C-GTP, indicating again that the maximum UCST is about 2.5 (see Fig. 9). To corroborate our phase behavior predictions based upon chain collapse, direct phase equilibria calculations using the EG (Ref. 28) method are now discussed.

\section{Phase equilibria calculations}

Figure 10 shows the fluid-fluid phase boundary in the temperature-composition (weight fraction) plane for chains of size 20 at reduced pressures of 0.15 and 0.2 . (The reduced critical pressure of the pure monomeric solvent is 0.10.) The corresponding reduced critical solution temperatures are estimated to be $1.16 \pm 0.03$ and $1.26 \pm 0.03$, respectively. Since the reduced critical temperature of the monomeric solvent is 1.08 , these phase boundaries are in the supercritical solvent region. For very long chains one would expect the LCST to drop into the subcritical region.

Remarkably, the intersection of the solvent equation of state with the C-GTT and C-GTP, defined for the quasi-ideal single chain and observed in Figs. $6-8$, is the signature of the LCST as seen in Fig. 10 for $P^{*}=0.15$ and 0.20 . Table I shows that the predicted LCST from the chain conformation studies and the direct phase behavior simulations are in excellent agreement at $P^{*}=0.15$ and 0.20 . Note that only single chain conformations (infinite dilution) were used to predict the full $P-T$ behavior of a finite-concentration poly-

TABLE I. Comparison between the predicted LCST from chain collapse information and the calculated LCST from direct phase equilibria simulations using the expanded Gibbs (EG) ensemble method.

\begin{tabular}{ccc}
\hline \hline $\begin{array}{c}\text { Pressure } \\
P \sigma^{3} / \epsilon\end{array}$ & $\begin{array}{c}\text { LCST from chain } \\
\text { collapse studies }\end{array}$ & $\begin{array}{c}\text { LCST from } \\
\text { EG simulations }\end{array}$ \\
\hline 0.15 & $1.13^{\mathrm{a}}$ & $1.16 \pm 0.03$ \\
0.20 & $1.21^{\mathrm{a}}$ & $1.26 \pm 0.03$ \\
\hline \hline
\end{tabular}

${ }^{a}$ These estimations are interpolations from the C-GTT in Figs. 7 and 8 , hence no errors are reported.

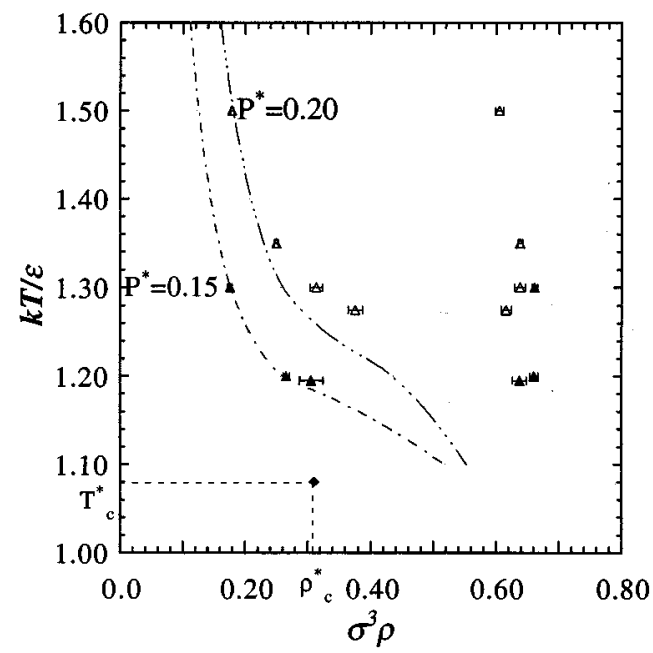

FIG. 11. Temperature-solution density phase diagram at the indicated pressures. Dashed lines denote pure solvent isobars at $P^{*}=0.20$ and $P^{*}$ $=0.15$. Vapor-liquid critical point for the pure solvent is denoted by the filled diamond.

mer solution (see Fig. 9). The single chain simulations are much simpler and less computationally expensive than direct phase equilibria simulations.

Figure 11 shows the LCST phase behavior on a temperature versus density phase diagram. Note that the pure solvent isobars (dashed lines) are relatively close to the polymer-lean branch of the phase diagram. At any temperature and pressure, the monomeric solvent always has a lower density (monomers per unit volume) than the pure chain liquid. This is also true in the absence of attractive interactions. At fixed pressure and in the normal liquid range for the solvent, this density difference between pure solvent and pure polymer increases with increasing temperature because the polymer has a smaller thermal expansion coefficient. Adding dense, pure polymer to less-dense, pure solvent yields a solution whose density is intermediate between that of the solvent and polymer. The final solution density is established by opposing thermodynamic forces. A favorable low potential energy is caused by high density, because this increases the strength of favorable chain-solvent attractive interactions, but system entropy is increased by lowering density (larger volume). Below the LCST, in the single phase region of the phase diagram, energetics wins the battle, and the final density is such that there is a net volume contraction. ${ }^{7,47}$ That is, formation of a homogeneous single phase requires a negative volume change (entropically unfavorable) with an associated negative heat of mixing (energetically favorable). Note that the observed increase of the LCST with pressure is consistent with a negative volume of mixing. ${ }^{7}$ Overall, the mixing process is favorable, despite the small loss of entropy associated with the volume contraction. However, the volume contraction grows with increasing temperature and the associated entropy loss increases. Finally, the entropic penalty required to form a single phase becomes too severe and the solution splits into two fluid phases; a dense polymer-rich 
phase and less-dense polymer-depleted phase. The total free energy of the phase separated system is less than that of a single phase system with the same overall composition. (The overall volume of the phase separated system will also be larger than that of the single phase system.) Most of the polymer will partition into the dense solvent phase to optimize solvent-chain energetic interactions; a much smaller amount of polymer is found in the less-dense solvent phase (see Fig. 11). The dense polymer-rich phase can be thought of as the energetically favorable phase, while the less-dense solvent phase is the more entropically favorable phase. The polymer-rich branch of the phase diagram in Figs. 10 and 11 is the high density solvent phase, whereas the polymer-dilute branch is the low density solvent phase.

\section{Nature of the UCST and LCST}

It is well-known that an UCST is caused by unfavorable energetics. The system lowers its potential energy by phase separating. For symmetric energetics, there are no unfavorable interactions. $\left(\epsilon_{11}=\epsilon_{22}=\epsilon_{12} \Rightarrow\right.$ classical interaction parameter, $\chi=0$.) But an energetic mechanism that could drive phase separation is still available in a compressible system. At temperatures well above the LCST, the solvent density is very low and the potential energy of the system is very high. The potential energy can be lowered if a 2 phase system forms in which one phase is of higher density than the single phase system. Entropically, the dense phase is not favorable relative to the single phase system, but energetically it is favorable because attractive interactions are enhanced. The total system potential energy can be lowered by phase splitting under these conditions of very low solvent density. That is, the phase separation would be energetically driven, consistent with the observation of an UCST. A hint of the possibility of a closed immiscibility loop in the phase diagram is also seen in Fig. 11 at the highest temperature $\left(T^{*}=1.5\right)$. Indeed, the research group at the University of Wisconsin has already corroborated the existence of the closed immiscibility loop and it will be subject of a forthcoming paper. ${ }^{33}$

Phase instability near the vapor-liquid critical point of the solvent is more transparent from the isothermal stability condition, ${ }^{7}$

$$
\left(\frac{\partial^{2} g}{\partial \phi^{2}}\right)_{P}=\left(\frac{\partial^{2} a}{\partial \phi^{2}}\right)_{\rho}-\frac{\beta}{\rho}\left(\frac{\partial P}{\partial \phi}\right)_{\rho}^{2}>0,
$$

where $g$ and $a$ are the intensive Gibbs and Helmholtz free energies (per monomer of solution), $\beta$ is the isothermal compressibility of the solution, $\rho$ is the solution monomer density (monomers per unit volume), and $\phi$ is the occupied volume fraction of either component. The "incompressible contribution," $\left(\partial^{2} a / \partial \phi^{2}\right)_{\rho}$, is dominated by the classical entropy of mixing and is expected to be positive for the symmetrical energetics employed in this study. (A solution is in general more random than its pure components; therefore, the classical entropy of mixing is inherently positive.) The second term, the "compressible contribution," is scaled by the solution compressibility and always contributes unfavorably to phase stability. If the solution is primarily solvent, the com- pressibility becomes very large as the solvent liquid-vapor critical point is approached; the solution becomes unstable and splits into two fluid phases. The pressure derivative, $(\partial P / \partial \phi)_{\rho}$, is given approximately by

$$
\left(\frac{\partial P}{\partial \phi}\right)_{\rho}^{2} \cong\left[P_{1}(T, \rho)-P_{2}(T, \rho)\right]^{2},
$$

where the subscripts 1 and 2 on $P$ refer to solvent and polymer, respectively. These 2 pressures, $P_{1}$ and $P_{2}$, are the hypothetical pressures of the pure solvent and pure polymer at the same temperature and monomer density $\rho$ as the solution. Negative pressures for the polymer are usually required, since $\rho_{1}<\rho<\rho_{2}$ at the system pressure $P$; $\rho_{1}$ is the pure solvent monomer density and $\rho_{2}$ is the pure polymer monomer density at the system pressure $P$. The $P V T$ properties of the two pure components affect solution thermodynamics primarily through this term, $(\partial P / \partial \phi)_{\rho}$. Thus, it is clear that finite compressibility and equation of state differences between components play an important role in solution phase behavior.

The simulation results for polymer conformation and phase equilibria provide insight into experimental studies of polymer-SCF LCST phase behavior ${ }^{14,16}$ and of flocculation of emulsions and latexes stabilized by block-copolymers in SCFs. For example, O'Neill et al. ${ }^{21}$ determined the density at the onset of phase separation for a dilute poly(1,1dihydroperfluorooctyl acrylate) (PFOA) solution in compressed $\mathrm{CO}_{2}$. For a reduced temperature, $T_{r}=T / T_{c}$ of 1.05 , the reduced theta-density, $\rho_{c}$, was 1.7. The cohesive energy density of this polymer is extremely low as indicated by its very low surface tension of $10 \mathrm{dyn} / \mathrm{cm}$. Consequently, its cohesive energy density is closer to that of $\mathrm{CO}_{2}$ than that of nearly all other polymers. From an energetic point of view, this system is relatively symmetric, and thus it is reasonable to compare it with the simulations in this study. At this same $T_{r}=1.05$, the experimental LCST occurs at a similar reduced density as the C-GTT (from Fig. 7), in close agreement with experiment (see Table II).

Polymer chain collapse plays an important role in the flocculation of emulsions and latexes stabilized by blockcopolymers. Lattice-fluid self-consistent field (LFSCF) theory has been used to describe the interaction between two surfaces containing grafted or adsorbed polymeric stabilizers. ${ }^{48,49}$ As the solvent density is lowered, solvent molecules leave the polymer chains to raise the volume and entropy of the system. The polymer chains collapse toward the surface. This separation of polymer and solvent is analogous to LCST phase separation in bulk. Recently, O'Neill et al. $^{21}$ and Yates et al. ${ }^{22}$ used turbidimetry and dynamic light scattering to measure critical flocculation densities (CFD) for emulsions stabilized with polystyrene$b$-poly(FOA). The experimental value of the critical flocculation density was closely related to the theta density for the PFOA block (Table II). In conclusion, the CFD from experiment and LFSCF theory and the C-GTT and LCST from our simulations all occur at about the same density. Thus chain 
TABLE II. Comparison among experiments (Refs. 21, 22), lattice-fluid selfconsistent field (LFSCF) (Ref. 49) theory, and simulations for the onset of phase separation for a bulk polymer solution and for a stabilized emulsion in a compressed fluid mixture at $T_{r}=T / T_{c}=1.05$.

\begin{tabular}{lc}
\hline \hline \multicolumn{1}{c}{ System } & $\begin{array}{c}\text { Onset of phase separation } \\
\left(\rho / \rho_{c}\right)\end{array}$ \\
\hline $\begin{array}{l}\text { PFOA }^{\mathrm{a}} \text { in compressed } \mathrm{CO}_{2} \\
\quad \text { (experiment) }^{\mathrm{c}}\end{array}$ & $1.7^{\mathrm{b}}$ \\
$\begin{array}{l}\text { CFD from turbidimetry; emulsion } \\
\text { stabilized with PS-b-PFOA } \\
\quad \text { (experiment) }^{\mathrm{c}}\end{array}$ & 1.7 \\
$\begin{array}{l}\text { LFSCF theory } \\
\text { bulk stabilizer phase behavior }\end{array}$ & 1.5 \\
$\begin{array}{l}\text { CFD from LFSCF theory, } \\
\text { emulsion+stabilizer }\end{array}$ & 1.5 \\
$\begin{array}{l}\text { Chain collapse simulation of a } \\
\text { Lennard-Jones system } \\
\text { (this study) }\end{array}$ & 1.5 \\
\hline \hline
\end{tabular}

${ }^{a}$ See text for chemical name.

${ }^{\mathrm{b}}$ This value is from an extrapolation to infinite polymer molecular weight, which represents the theta-density.

${ }^{c}$ References 21 and 22 .

${ }^{\mathrm{d}}$ Reference 49.

collapse plays a central role in polymer solvent phase separation and in the stability of colloidal interactions.

\section{CONCLUSIONS}

The chain conformation calculations presented in this study give insight into the rich density-dependent behavior of homopolymers in compressible solvents. At high solvent densities, the chain adopts coil-like conformations that approach the athermal or infinite temperature limit. As the solvent density is reduced isothermally, the loss in solvent quality causes chain collapse. This result is opposite to that observed in athermal systems without attractive interactions, where an isolated chain is expanded at low solvent density and gradually collapses as density is increased (solvent-chain excluded volume effect). Attractive interactions seem to dominate any excluded volume effects and completely change the density dependence of chain conformation.

In vacuum, the dimensions of an isolated chain are determined by two opposing forces, attractive energetics and entropy. Upon cooling, a coil-to-globule transition occurs at a unique temperature (C-GTT) where attractive and selfexcluded volume forces are balanced. The C-GTT, defined at the point where $\mathfrak{N}=\left\langle R^{2}\right\rangle / 6\left\langle R_{g}^{2}\right\rangle=1$, was determined for a wide range of solvent densities in the gas, supercritical fluid, and liquid states. This is strictly an operational definition of the transition temperature and does not correspond to the usual definition of the theta temperature. However, as $N \rightarrow \infty$ we expect both definitions to coincide. When solvent molecules are present in the system, the C-GTT is depressed relative to its value in vacuum in a surprisingly simple nearlinear fashion. The primary effect of solvent is to mediate the intrachain attractive forces. In other words, the presence of the solvent near the chain impedes collapse by excluding close intrachain contacts (a solvent excluded volume effect). The denser the solvent, the more effective it is in screening attractive intrachain forces.

The most important result is the relationship between the single chain conformation and the phase boundary of a finite-concentration polymer solution. For a given pressure, the LCST from direct simulation of mixtures is very close to the C-GTT for a single chain. The balance of binary excluded volume and attractive interactions for a single quasiideal chain at the C-GTT signals the onset of phase transition in a polymer solution near the LCST. At temperatures below the LCST, the chains exist in expanded coillike conformations at all solution compositions. Just above the LCST and in the two phase region, chains are still expanded on the polymer-rich branch (or the high-density branch) of the phase diagram, but the chains are partially collapsed on the dilute-polymer (or low-density) branch of the phase diagram. At temperatures well above the LCST, the chains begin to expand again. This suggests the existence of a closed immiscibility loop with an UCST at very high temperatures above the LCST, as has been demonstrated with direct simulations of phase separation with a novel expanded Gibbs ensemble (EG) method. ${ }^{33}$ The simulation results for both polymer conformation and phase equilibria provide insight into experimental studies of polymer-supercritical fluid LCST phase behavior and critical flocculation density (CFD) of emulsions and latexes stabilized by block-copolymers. For a symmetric polymer-solvent system, the CFD and LCST from experiment ${ }^{21,22}$ and theory ${ }^{49}$ occur at about the same reduced density as the LCST and C-GTT from these simulations.

\section{ACKNOWLEDGMENTS}

K.P.J. acknowledges support from the NSF (CTS9626826, CTS-9358406), DOE (DEFG03-96ER14664), the Separations Research Program at the University of Texas, and the Texas Advanced Research Program. J.J.dP. recognizes NSF Grant No. CTS-9629135. I.C.S. recognizes financial support from the NSF and the Texas Advanced Research Program. J.C.M. recognizes the Eastman Chemical Company for a graduate research fellowship. We thank Kent Milfeld for many helpful discussions and the UT High Performance Computing Facility for a Cray J90 Time Grant.

\footnotetext{
${ }^{1}$ C. Williams, F. Brochard, and H. L. Frisch, Annu. Rev. Phys. Chem. 32, 433 (1981).

${ }^{2}$ W. H. Stockmayer, Macromol. Chem. 35, 54 (1960).

${ }^{3}$ I. C. Sanchez and D. J. Lohse, Macromolecules 14, 131 (1981).

${ }^{4}$ A. Milchev, W. Paul, and K. Binder, J. Chem. Phys. 99, 4786 (1993).

${ }^{5}$ A. M. Rubio, J. J. Freire, J. H. R. Clarke, C. W. Yong, and M. Bishop, J. Chem. Phys. 102, 2277 (1995).

${ }^{6}$ R. Perzynski, M. Delsanti, and M. Adam, J. Phys. (Paris) 45, 1765 (1984).

${ }^{7}$ I. C. Sanchez, Encyclopedia of Physical Science and Technology (Academic, New York, 1992), Vol. XIII, p. 153.

${ }^{8}$ B. E. Eichinger and P. J. Flory, Trans. Faraday Soc. 64, 2035 (1968).

${ }^{9}$ I. C. Sanchez and R. H. Lacombe, Macromolecules 11, 1145 (1978).

${ }^{10}$ I. C. Sanchez, Macromolecules 24, 908 (1991).

${ }^{11}$ P. J. Flory, Principles of Polymer Chemistry (Cornell University Press, Ithaca, 1953).

${ }^{12}$ I. C. Sanchez, Macromolecules 12, 980 (1979).

${ }^{13}$ M. A. Meilchen, B. M. Hasch, and M. A. McHugh, Macromolecules 24, 4974 (1991).
} 
${ }^{14}$ S. Mawson, K. P. Johnston, J. R. Combes, and J. M. DeSimone, Macromolecules 28, 3182 (1995).

${ }^{15}$ E. Kiran and W. Zhuang, Polymer 33, 5459 (1992).

${ }^{16}$ B. Folie and M. Radosz, Ind. Eng. Chem. Res. 34, 1501 (1995).

${ }^{17}$ A. V. Yazdi, C. Lepilleur, E. J. Singley, L. W. F. A. Adamsky, R. M. Enick, and E. J. Beckman, Fluid Phase Equilibria 117, 297 (1996).

${ }^{18}$ K. P. Johnston, K. L. Harrison, M. J. Clarke, S. M. Howdle, M. P. Heitz, F. V. Bright, C. Carlier, and T. W. Randolph, Science 271, 624 (1996).

${ }^{19}$ K. A. Bartscherer, H. Renon, and M. Minier, Fluid Phase Equilibria 107, 93 (1995).

${ }^{20}$ J. B. McClain, D. Londono, J. R. Combes, T. J. Romack, D. A. Canelas, D. E. Betts, G. D. Wignall, E. T. Samulski, and J. M. DeSimone, J. Am. Chem. Soc. 118, 917 (1996).

${ }^{21}$ M. L. O'Neill, M. Z. Yates, K. L. Harrison, K. P. Johnston, D. A. Canelas, D. E. Betts, J. M. DeSimone, and S. P. Wilkinson, Macromolecules 30, 5050 (1997).

${ }^{22}$ M. Z. Yates, M. L. O’Neill, K. P. Johnston, S. Webber, D. A. Canales, D. A. Betts, and J. M. DeSimone, Macromolecules 30, 5060 (1997).

${ }^{23} \mathrm{~K}$. Binder, Monte Carlo and Molecular Dynamics Simulations in Polymer Science (Oxford University Press, New York, 1995).

${ }^{24}$ J. J. dePablo, M. Laso, and U. W. Suter, J. Chem. Phys. 96, 2395 (1992).

${ }^{25}$ J. J. dePablo, M. Laso, U. W. Suter, and H. D. Cochran, Fluid Phase Equilibria 83, 323 (1993).

${ }^{26}$ A. Z. Panagiotopoulos, Mol. Phys. 63, 527 (1988).

${ }^{27}$ F. A. Escobedo and J. J. dePablo, Mol. Phys. 87, 347 (1996).

${ }^{28}$ F. A. Escobedo and J. J. dePablo, J. Chem. Phys. 105, 4391 (1996).

${ }^{29}$ G. Luna-Barcenas, D. G. Gromov, J. C. Meredith, I. C. Sanchez, J. J. dePablo, and K. P. Johnston, Chem. Phys. Lett. (in press).

${ }^{30}$ G. Luna-Bárcenas, G. E. Bennett, I. C. Sanchez, and K. P. Johnston, J. Chem. Phys. 104, 9971 (1996).
${ }^{31}$ J. K. Johnson, J. A. Zollweg, and K. E. Gubbins, Mol. Phys. 78, 591 (1993).

${ }^{32}$ B. Smit, J. Chem. Phys. 96, 8639 (1992).

${ }^{33}$ D. G. Gromov, Ph.D. dissertation, University of Wisconsin, 1997.

${ }^{34}$ E. Leontidis, J. J. dePablo, M. Laso, and U. W. Suter, Advances in Polymer Science (Springer, New York, 1994).

${ }^{35}$ S. K. Kumar, I. Szleeifer, and A. Z. Panagiotopoulos, Phys. Rev. Lett. 66, 2935 (1991).

${ }^{36}$ I. Szleifer and A. Z. Panagiotopoulos, J. Chem. Phys. 97, 6666 (1992).

${ }^{37}$ M. Laso, J. J. dePablo, and U. W. Suter, J. Chem. Phys. 97, 2817 (1992).

${ }^{38}$ S.-T. Sun, I. Nishio, G. Swislow, and T. Tanaka, J. Chem. Phys. 73, 5971 (1980).

${ }^{39}$ F. A. Escobedo and J. J. dePablo, Mol. Phys. 89, 1733 (1996).

${ }^{40}$ J. K. C. Suen, F. A. Escobedo, and J. J. dePablo (submitted).

${ }^{41}$ J. desCloizeaux and G. Jannink, Polymers in Solution: Their Modeling and Structure (Clarendon, Oxford, 1990).

${ }^{42}$ I. C. Sanchez, "Statistical properties of some model macromolecules," Dissertation, University of Delaware, 1969.

${ }^{43}$ C. Domb and F. T. Hioe, J. Chem. Phys. 51, 1920 (1969).

${ }^{44}$ T. A. Witten and L. Schafer, J. Phys. A 11, 1843 (1985).

${ }^{45}$ M. Rawiso, R. Duplessix, and C. Picot, Macromolecules 20, 630 (1987).

${ }^{46}$ G. S. Grest and M. Murat, Macromolecules 26, 3108 (1993).

${ }^{47}$ I. C. Sanchez, Polymer Compatibility and Incompatibility: Principles and Practices, MMI Symposium Series, edited by K. Solc (MMI, New York, 1982), Vol. 2, p. 59

${ }^{48}$ D. G. Peck and K. P. Johnston, Macromolecules 26, 1537 (1993).

${ }^{49}$ J. C. Meredith and K. P. Johnston (in preparation). 